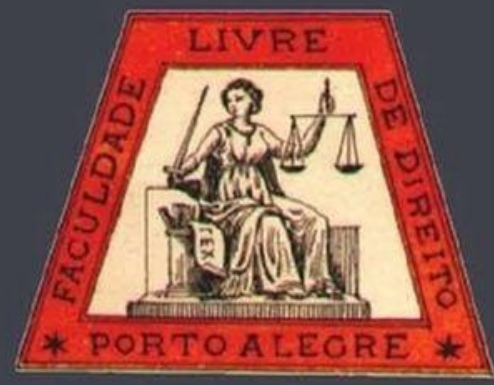

\title{
Autorregulação e riscos: desafios e possibilidades jurídicos para a gestão dos resíduos nanoparticulados
}

Self-regulation and risks: legal challenges and possibilities for nanoparticulate waste management

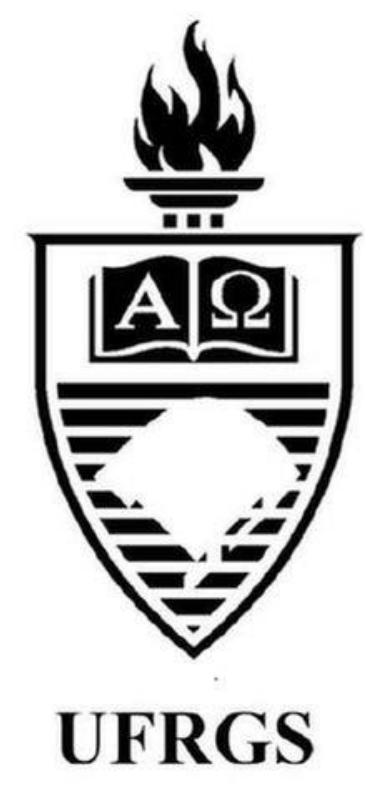

Wilson Engelmann

Universidade do Vale do Rio dos Sinos

Raquel Von Hohendorff

Universidade do Vale do Rio dos Sinos

Daniele Weber S. Leal

Universidade do Vale do Rio dos Sinos 


\section{Autorregulação e riscos: desafios e possibilidades jurídicos para a gestão dos resíduos nanoparticulados}

Self-regulation and risks: legal challenges and possibilities for nanoparticulate waste management

Wilson Engelmann ${ }^{*}$

Raquel Von Hohendorff ${ }^{* *}$

Daniele Weber S. Leal ${ }^{* * *}$

\section{REFERÊNCIA}

LEAL, Daniele Weber S.; ENGELMANN, Wilson; HOHENDORFF, Raquel Von. Autorregulação e riscos: desafios e possibilidades jurídicos para a gestão dos resíduos nanoparticulados. Revista da Faculdade de Direito da UFRGS, Porto Alegre, n. 39, vol. esp., p. 211-232, dez. 2018.

\section{RESUMO}

A utilização industrial da escala nanométrica está avançando rapidamente sem que se tenha uma certeza científica sobre a segurança das nanopartículas e sem que a área jurídica tenha construído marco regulatório específico. As nanotecnologias vêm acompanhadas de incertezas científicas quanto seus efeitos e (possíveis?) danos futuros ao meio ambiente e vida humana. Com o aumento do consumo destes produtos, amplia-se também o descarte de nanomateriais no meio ambiente. Assim, a destinação final dos nanomateriais desperta uma enorme incógnita e clama por possíveis soluções. De que forma deveria ocorrer a destinação final do resíduo nanotecnológico? O Sistema do Direito vem enfrentando as complexidades do risco? E de que maneira o Direito poderá dar respostas adequadas para a gestão do nanowaste? $\mathrm{O}$ problema que se pretende enfrentar neste artigo é: uma vez que inexiste regulação específica sobre o nanowaste, de que forma seria viável a utilização do protocolo da OCDE? Baseado na cautela frente à incerteza, uma resposta adequada seria a adoção de algum instrumento regulatório, mesmo que estranho ao Direito, fundamentado no pluralismo jurídico de Teubner, respeitando a precaução e buscando evitar possível dano ambiental e às futuras gerações.

PALAVRAS-CHAVE

Nanotecnologias. Riscos. Resíduos. Autorregulação.

\section{ABSTRACT}

Industrial use of the nanometer scale is advancing rapidly regardless of the absence scientific certainty about the safety of nanoparticles and without the legal area having built a specific regulatory framework. Nanotechnologies are accompanied by scientific uncertainties as to their effects and (possible?) future harm to the environment and human life. With the increase in consumption of these products, the disposal of nanomaterials to the environment is also increasing. Thus, the final destination of nanomaterials arouses a huge unknown and calls for possible solutions. How should the final disposal of the nanotechnology residue take place? Is the Law System facing the complexities of risk? And how can the Law provide adequate responses to the management of nanowaste? The problem to be addressed in this article is the following: since there is no specific regulation on nanowaste, how would the use of the OECD protocol be feasible? Based on caution in the face of uncertainty, an adequate response would be the adoption of some regulatory instrument, even if unfamiliar to the Law, based on Teubner's legal pluralism, respecting precaution and seeking to avoid possible environmental damage to the future generations.

\section{KEYWORDS}

Nanotechnologies. Risks. Waste. Self-regulation.

\footnotetext{
* Professor e Coordenador Adjunto do Programa de Pós-Graduação em Direito da Universidade do Vale do Rio dos Sinos. Doutor em Direito pela Universidade do Vale do Rio dos Sinos (UNISINOS).

${ }^{* *}$ Doutora pelo PPGD da Universidade do Vale do Rio dos Sinos (UNISINOS).

**** Mestre pelo PPGD da Universidade do Vale do Rio dos Sinos (UNISINOS).
} 


\section{SUMÁRIO}

Introdução; 1. Desvelando as nanotecnologias: o debate global sobre novos riscos. 2. Nanoprodutos e nanowaste: uma nova "escala" de riscos?. 3. Alternativas (auto)regulatórias para gestão do risco: flexibilização do Sistema do Direito? Conclusão. Referências.

\section{INTRODUÇÃO}

O mundo na escala nano sempre existiu integrando a natureza, mas, somente a partir da metade para o final do século XX, o ser humano conseguiu acessar esta ordem de grandeza, visualizando a bilionésima parte de um metro. Observa-se no cotidiano da vida humana o consumo cada vez maior de inúmeros novos produtos com nanotecnologia, nas mais diversas áreas.

Como exemplo podem ser citados protetores solares, calçados, telefones celulares, tecidos, cosméticos, automóveis, medicamentos produtos para agricultura, medicamentos veterinários, produtos para tratamento de água, materiais para a construção civil, plásticos e polímeros, produtos para uso nas indústrias aeroespacial, naval e automotora, siderurgia, entre outros.

Assim, deixam de ser apenas promessas futurísticas e incorporam-se na rotina diária da sociedade deste início do século XXI, exigindo, portanto, a atenção por parte do Sistema do Direito. Mas as nanotecnologias vêm acompanhadas de incertezas científicas quanto seus efeitos e danos futuros ao meio ambiente e vida humana. Uma vez que o consumo destes produtos vem sendo cada vez maior, amplia-se também o aporte destes materiais ao meio ambiente. Desta forma, a questão nanolixo ou nanorresíduo é mais um aspecto a gerar preocupações. Trata-se da incógnita do nanowaste. De que forma deveria ocorrer a destinação final do resíduo nanotecnológico? Há diferença dos resíduos em escala macro?
Ainda, face a inexistência de regulação específica das nanotecnologias, tampouco sobre o nanowaste, é necessário buscar uma alternativa para implementação de instrumentos com potencial regulatório (mesmo que elaborados fora do mundo jurídico). Muitos estudos sobre as nanotecnologias estão em desenvolvimento em nível mundial, como nos Estados Unidos, na Europa em geral, na Ásia e na América Latina. Organizações como ISO, NIOSH, FDA, NANOREG (Europa), British Standars Institution (BSI), a Comissão Europeia e Parlamento Europeu, e por fim, a OCDE vêm desenvolvendo estudos, protocolos e instrumentos com potencial regulatório, que poderiam (ou não) fazer as vezes de marco regulatório, com o objetivo de sanar a lacuna legislativa e omissão estatal.

Para tanto, utilizar-se-á a perspectiva metodológica sistêmico-construtivista, a partir das contribuições de Niklas Luhmann, Gunther Teubner, que considera a realidade como uma construção de um observador, analisando todas as peculiaridades implicadas na observação, tratando-se de uma forma de reflexão jurídica sobre as próprias condições de produção de sentido, bem como as possibilidades de compreensão das múltiplas dinâmicas comunicativas diferenciadas em um ambiente complexo, como é o gerado pelas nanotecnologias. Utilizar-se-á a pesquisa bibliográfica, buscando-se realizar a revisão das publicações em livros, artigos científicos e sítios oficiais da internet, alinhada à metodologia sistêmica-construtivista, utilizando-se de bases que não compõem o Direito tradicional (que pode ser entendida como legislação ou normas positivadas), possibilitando a conexão e aplicação 
de outros institutos regulatórios, realizando a interligação de outras áreas da ciência, como a engenharia, química, engenharia ambiental, engenharia de materiais e produtos, medicina, dentre outras.

É necessário que se parta de conhecimentos prévios acerca das nanotecnologias e seu debate em nível internacional, bem como a questão do risco; ademais, analisar-se-á o ciclo de vida dos nanomateriais e atenção necessária ao nanowaste; e por fim, conhecer o estudo específico sobre nanowaste da OCDE, e de que maneira será viável a utilização de tal instrumento com potencial regulatório, realizando a necessária gestão do risco. Portanto, o problema que se pretende enfrentar neste artigo, poderá ser assim circunscrito: uma vez que inexiste regulação específica sobre o nanowaste, de que forma seria viável a utilização do protocolo da OCDE? Quais teorias possibilitam a adoção de instrumentos com potencial regulatório que versam sobre os resíduos nanotecnológicos?

Considerando-se a necessária cautela frente à incerteza, uma resposta adequada seria a adoção de um instrumento regulatório, mesmo que não proveniente do mundo jurídico tradicional, objetivando o respeito à precaução, à sustentabilidade e à equidade intergeracional e o fornecimento de respostas adequadas à complexidade que se apresenta.

\section{DESVELANDO}

AS

\section{NANOTECNOLOGIAS: O DEBATE GLOBAL SOBRE NOVOS RISCOS}

$O$ século XXI se caracteriza pela emergência de uma revolução tecnocientífica sem precedentes, impulsionada pelos avanços de novos produtos e dispositivos com nanotecnologias. $\mathrm{O}$ número de produtos à base de nanotecnologias cresce rapidamente no mercado consumidor: de acordo com o Banco de dados de produtos com nanotecnologia (Nanotechnology
Products Database) (NPD, 2018), em dados atualizados até julho de 2018, existem 8.242 produtos que têm algum componente à base de nanotecnologia, produzidos em 1961 empresas, localizadas em 56 países. Segundo essa mesma base de dados, os principais setores onde se encontram os produtos são alimentos, cosméticos, eletrônicos, equipamentos domésticos, petróleo, energias renováveis, têxteis, medicina, indústria ambiental, como água, remedição e purificação de água, tratamento de água residual e purificação de solo, além de artigos esportivos e fitness. Verifica-se uma grande versatilidade na aplicação da escala nano, o que lhe garante o sucesso e o crescimento rápido e em um curto espaço de tempo. Como destaca Klaus Schwab (2016), as nanotecnologias estão no centro da estruturação da denominada quarta revolução industrial.

Provavelmente, o fascínio da criatividade levou os cientistas a desenvolver condições técnicas para pesquisar nesta escala, desvendando um "mundo novo", com possibilidades e riscos ainda em grande parte desconhecidos. Apesar disso, a utilização industrial da escala nanométrica está avançando rapidamente, incrementando-se os produtos desenvolvidos à base da nano escala, sem que se tenha uma certeza científica sobre a segurança das nanopartículas e sem que a área jurídica tenha construído marco regulatório específico. Em grande parte, este cenário está circunscrito à busca da inovação e a necessidade de se transferir o conhecimento científico gerado nos laboratórios das Universidades e Centros de Pesquisa para o setor industrial, que transforma a pesquisa em produtos.

O tema das nanotecnologias integra a Estratégia do Programa Quadro "Horizonte 2020" da União Europeia, estabelecendo expressamente: As tecnologias facilitadoras essenciais, como, por
exemplo, a indústria de ponta e de materiais
avançados, a biotecnologia e as nanotecnologias,
estão no cerne dos produtos inovadores: telefones
inteligentes, baterias de alto rendimento, veículos 
ligeiros, nanomedicamentos, tecidos inteligentes e muito mais. A indústria transformadora europeia é o maior empregador, com 31 milhões de trabalhadores em toda a Europa (HORIZON 2020, 2017).

Além disso, cabe destacar o documento intitulado: Principais tecnologias facilitadoras, do grupo de especialistas de alto nível (High-Level Expert Groupon Key Enabling Technologies) (2015), referente a uma comunicação da Comissão Europeia datada de 2009, intitulada "Preparando-se para o nosso futuro: desenvolvendo uma estratégia comum para tecnologias facilitadoras essenciais na União Europeia"(Preparing for our future: developing a Common Strategy for Key Enabling Technologies in the EU). Neste documento foram identificadas seis tecnologias facilitadoras, e uma delas são as nanotecnologias.

O campo das nanotecnologias está avançando rapidamente e deverá impactar em praticamente todas as facetas globais da indústria e da sociedade. A padronização internacional em nanotecnologias deve contribuir para que se perceba o potencial desta tecnologia através do desenvolvimento econômico, melhoria da qualidade de vida e para a melhora e proteção da saúde pública e do meio ambiente. Pode-se esperar que muitos nanomateriais recémfabricados entrem no mercado e nos locais de trabalho (ISO, 2017). Esta rápida transição dos laboratórios para as fábricas e, a partir da produção em grande escala, para o mercado consumidor, reside um importante foco de possibilidade de riscos não adequadamente avaliados e conhecidos. Vale dizer: é uma face a ser observada pelo sistema do Direito. Quando se utiliza a expressão sistema, faz-se uma alusão à obra de Luhmann, já que o Direito, observado dentro de um sistema de normas, torna-se complexo, não conseguindo prontamente estabelecer respostas e comunicação entre seus elementos, e assim, promove sua mutação pelas irritações (LUHMANN, 2005). (LUHMANN, 1990).

Desta forma, a quantidade de produtos aumenta rapidamente, e paralelamente a esse desenvolvimento crescem as preocupações sobre os riscos que as nanotecnologias poderão provocar, especialmente em relação aos trabalhadores, em virtude de sua exposição, devendo trazer-se o foco para a relação com o meio ambiente, saúde e segurança (ENGELMANN, 2011, p. 342).

Neste panorama, destacam-se diversas características das nanotecnologias, tais como as propriedades incomuns de nanopartículas são principalmente baseadas em seu tamanho em nano escala e sua área de superfície. À medida que o tamanho de uma partícula diminui e se aproxima da nano escala, muitas propriedades começam a mudar em comparação com o mesmo material no seu tamanho macro. Cita-se, como exemplo, a cor e a temperatura de fusão do ouro, as quais são muito diferentes em nano escala que em ouro convencional. Os efeitos tóxicos de materiais que se mostram como inertes na escala macro, também são muito diferentes na escala nano. Como a área de superfície de partículas aumenta uma maior proporção dos seus átomos ou moléculas começar a ser exibida na superfície, em vez de o interior do material. Existe uma relação inversa entre o tamanho das partículas e o número de moléculas presente na superfície da partícula. $\mathrm{O}$ aumento na área de superfície determina o número potencial de grupos reativos sobre a partícula. A alteração das propriedades físicoquímicas e estruturais das nanopartículas com uma diminuição do tamanho poderá ser responsável por uma série de interações materiais que podem levar a efeitos toxicológicos.

Como as nanopartículas são muito pequenas, medindo menos de um centésimo de bilionésimo de metro, são regidas por leis físicas muito diferentes daquelas com as quais a ciência está acostumada. Existem probabilidades de que 
as nanopartículas apresentem grau de toxicidade maior do que as partículas em tamanhos normais, podendo assim ocasionar riscos à saúde e segurança de pesquisadores, trabalhadores e consumidores (HOHENDORFF, 2018, p.26).

De qualquer modo, se constatam três características que merecerão a atenção daqui para frente, inclusive por parte do Direito: a) velocidade: ao contrário das revoluções industriais anteriores, esta evolui em um ritmo exponencial e não linear ${ }^{1}$; b) amplitude e profundidade: ela tem a revolução digital como base e combina várias tecnologias, levando a mudanças de paradigma sem precedentes da economia, dos negócios, da sociedade e dos indivíduos; c) impacto sistêmico: ela envolve a transformação de sistemas inteiros entre países e dentro deles, em empresas, indústrias e em toda sociedade (SCHWAB, 2016, p.11).

Além destes aspectos, a apresentação de novidades trazidas pela pesquisa ou desenvolvimento de novos produtos sempre acarreta um certo nível de risco. ${ }^{2}$ Para o Direito, gera a necessidade de normatizar os riscos e os danos futuros com criatividade e modelos

\footnotetext{
${ }^{1}$ A noção sobre a diferença entre o crescimento linear e o crescimento exponencial, em termos de tecnologia, é muito importante: "Alguém que entende muito bem o conceito é o inventor, futurista, autor e diretor de engenharia do Google, Ray Kurzweil (que a Revista Forbes descreve como 'a melhor máquina pensante' - isso é um apelido muito legal). Kurzweil usa uma analogia de, primeiramente, tomar 30 passos lineares (onde a distância de cada passo é a mesma que a anterior), e então tomar 30 passos exponenciais (onde a distância de cada passo é o dobro da anterior). A distância percorrida em 30 passos lineares é de aproximadamente 20 a 30 metros (dependendo do comprimento das pernas). Isso pode levá-lo de um lado da sua casa para o outro, ou através de uma rua movimentada da cidade. No entanto, tomar 30 passos exponenciais o leva ao redor do mundo 25 vezes (uma distância de cerca de um milhão de quilômetros) ou para a lua e vice-versa". (Someone who does understand the concept pretty well is inventor, futurist, author and director of engineering at Google, Ray Kurzweil (whom Forbes Magazine describes as 'the ultimate thinking machine' that's a pretty cool moniker). Kurzweil uses an analogy of firstly taking 30 linear steps (where the distance of each step is the same as the last), and then taking 30 exponential steps (where the distance of each step is double that of the
}

flexíveis, que possam ser facilmente modificados e atualizados em atendimento ao mesmo movimento das atualidades nanotecnológicas. $\mathrm{O}$ que é um grande desafio, pois o Direito sempre avaliou os fatos do passado, atribuindo-lhes efeitos jurídicos no presente, determinando como deveria ser o comportamento no futuro. No caso das nanotecnologias, os efeitos que poderão surgir - riscos e danos - no futuro e no presente, ainda são pouco conhecidos ${ }^{3}$. E esse também é o problema da regulação legislativa estatal sobre a matéria: ainda não se tem informações científicas suficientes para uma regulação formal.

É imperativo, portanto, que fabricantes (a indústria), laboratórios, cientistas e universidades, seguradoras e a comunidade jurídica, em ação conjunta na tomada de ações proativas, adotem medidas na identificação e quantificação dos riscos da nanotecnologia, bem como na efetivação de legislação de biossegurança e do adequado descarte do nanowaste (resíduos nanotecnológicos). Conhecer os riscos e adotar medidas precaucionais, através de protocolo adequado, é efetiva gestão do risco, e necessário

previous). The distance travelled by taking 30 linear steps is approximately 20 to 30 meters (depending on the length of your legs). This might get you from one end of your house to the other, or across a busy city street. However, taking 30 exponential steps gets you around the world 25 times (a distance of about a million kilometers) or to the moon and back again) (HOLLINGWORTH, 2016, p. 39-40).

${ }^{2}$ Segundo Andrea Signorino Barbat: “[...] É claro que qualquer novo empreendimento, em primeiro lugar, pode representar riscos especialmente até a plena compreensão e aprendizado de todos os aspectos envolvidos nele, seja pelo próprio comportamento da novidade ou por suas repercussões e interações com o exterior, objetos (meio ambiente) e temas (saúde, segurança, bem-estar)".(queda claro que todo emprendimiento novedoso, en primer término, puede suponer riesgos en especial hasta la cabal comprensión y aprendizage de todos los aspectos involucrados en él, ya sea por el comportamiento mismo de la novedad o por sus repercusiones e interacciones con el exterior, objetos (medio ambiente) y sujetos (salud, seguridad, bienestar). [...]". (BARBAT, 2016, p. 213).

${ }^{3} \mathrm{O}$ ser humano está construindo um mundo perigoso, como afirma DELMAS-MARTY, em Libertés et sûreté dans un monde dangereux. Éditions du Seuil, Paris, 2010. 
partir do Sistema do Direito prover tais respostas adequadas.

\section{NANOPRODUTOS E NANOWASTE: UMA NOVA "ESCALA" DE RISCOS?}

Os riscos podem ser incertos e ocorrer apenas no futuro, mas a decisão precisa ser realizada no presente, através da utilização destas novas ferramentas surgidas pela incorporação da ideia de que o conhecimento não poderá mais ficar aprisionado nos limites herméticos de cada campo do saber.

Somente assim o Direito poderá desenvolver respostas adequadas às demandas surgidas em função da nova realidade gerada pelo uso e impactos da tecnociência (e assim, das nanotecnologias), conjugando o respeito ao ser humano e ao meio ambiente com a inovação e ampliação do conhecimento nas áreas das ciências duras. É preciso um Direito crítico, capaz de fazer leituras da realidade e apto a provocar as mudanças necessárias nesta realidade, sob pena de restar isolado das outras áreas do conhecimento, que se utilizarão dos espaços vazios deixados pelo Direito, para atuarem, inclusive em questões regulatórias.

Abre-se um espaço de consequências ainda pouco conhecidas. Para o Direito, gera a necessidade para normatizar os riscos e os danos futuros. Trata-se de um grande desafio, pois o Direito sempre avaliou os fatos do passado, atribuindo-lhes efeitos jurídicos no presente, determinando como deveria ser o comportamento no futuro.

No caso das nanotecnologias, os efeitos que poderão surgir - riscos e danos - no futuro, no presente ainda são pouco conhecidos. No presente, ainda se tem poucas notícias sobre danos provocados pelas nanotecnologias. Esse o problema da regulação legislativa estatal sobre a matéria: ainda não se tem informações científicas suficientes para uma regulação formal. Busca-se sinalizar quais seriam as possibilidades de regulação para esse cenário de riscos, muitos dos quais ainda desconhecidos, que projetam para o futuro uma eventualidade de ocorrência de danos. Algumas pesquisas revelam uma desconexão entre as pesquisas acadêmicas e a sua aplicação em escala industrial, o que abre novas possibilidades para riscos.

Diferentemente dos riscos da modernidade, os riscos que resultam das novas tecnologias e do processo de industrialização são resultados de decisões conscientes (tomadas por um lado por organizações provadas e/ou estatais para obter vantagens econômicas e aproveitar as oportunidades correspondentes e, por outro lado, sobre a base de um cálculo que considera os perigos como a inevitável face oculta do progresso). São riscos distintos dos causados por catástrofes naturais e guerras, visto que se apresentam "por meios pacíficos, eles frutificam nos centros de racionalidade, ciência e bem-estar e desfrutam da proteção daqueles que cuidam da lei e da ordem" (BECK, 2008, p. 49).

$O$ crescente uso de materiais com nanotecnologia em produtos de consumo levantou certas preocupações sobre sua segurança para a saúde humana e o meio ambiente. Atualmente, há uma série de grandes incertezas e lacunas de conhecimento em relação ao comportamento, interações químicas e biológicas e propriedades toxicológicas destes materiais. Como lidar com essas incertezas exigirá a geração de novos conhecimentos básicos, é improvável que eles sejam resolvidos no futuro imediato (SOM, 2010), especialmente a partir das contribuições de apenas uma área do conhecimento. O exercício inter e/ou transdisciplinar será muito bem-vindo.

Portanto, um dos pontos que merece destaque, considerando o cenário analisado até o momento, se refere à juridicização dos riscos. Há evidências científicas de que os riscos poderão ser produzidos a partir de múltiplas fontes, como o risco de inalação e permanência profunda nos 
pulmões, acarretando danos por atravessar membranas biológicas(CHRISTENSEN, 2015); a comprovação de evidências de que os nanotubos de carbono são potencialmente perigosos em ambientes aquáticos, e que o mecanismo de toxicidade é complexo e insuficientemente compreendido até o momento (BRITTO, 2012); outro estudo mostra possíveis efeitos tóxicos no cérebro (neurotoxicidade) dos peixes Zebrafish (Danio rerio) expostos aos nanotubos de carbono (DAL FORNO et al., 2013). Apesar das promessas substanciais da nanotecnologia, se vislumbram várias áreas de preocupação, que motivam os atuais pedidos por regulação. Entre os possíveis riscos estão: a) a segurança do trabalhador na fabricação ou uso de partículas nanométricas; b) a segurança do consumidor na utilização ou aplicação de bens baseados na tecnologia nanotecnológica; c) danos ambientais causados pela produção de resíduos e fabricação de produtos acabados que possam contaminar o ar, a água ou o solo; d) convulsões socioeconômicas, incluindo rupturas econômicas em economias baseadas em agricultura, matériasprimas ou trabalho; e) consequências imprevistas de nanotecnologias descontroladas; f) uso governamental/estatal de nanotecnologias para conter as liberdades civis; g) aplicações militares; h) uso das nanotecnologias nas mãos de terroristas ou outros criminosos

(MARCHANT; SYLVESTER, 2006, 716).

A utilização das nanotecnologias sem uma avaliação adequada dos riscos e de uma gestão adequada destes riscos pode configurar-se em caminho como o do amianto, dos transgênicos e dos aerossóis, onde a comercialização passou muito à frente da avaliação ambiental dessas tecnologias.

Algumas das características que são úteis no desenvolvimento de produtos novos e melhorados também causam preocupação. Alguns nanomateriais são tipos inteiramente novos de composições, que podem ter novos e inesperados efeitos sobre os organismos vivos. O tamanho pequeno dos nanomateriais leva à preocupação sobre se eles podem atravessar membranas biológicas e, assim, ser tomadas por células e órgãos. Além disso, partículas pequenas podem se acumular profundamente nos pulmões se inaladas e permanecer lá por um longo período, porque eles são limpos lentamente a partir desta parte do pulmão. Existe também a preocupação de que o aumento da reatividade de materiais conhecidos na nanoforma pode levar a efeitos aumentados ou indesejados nos seres humanos e no ambiente (CHRISTENSEN, 2015, p. 334).

Os benefícios potenciais das nanotecnologias ainda não foram devidamente avaliados em relação ao potencial de riscos à saúde humana e ecológica. A análise do ciclo de vida dos produtos já tem sido reconhecida como uma ferramenta capaz de realizar uma avaliação ambiental, holística e assim, a pesquisa realizada por Gavankar e colaboradores (2012) demonstra que o número de estudos sobre o ciclo de vida de nanoprodutos vem aumentando, embora um olhar mais atento revela que muitos desses estudos não cobrem todo o ciclo de vida dos nanomateriais ou nanoprodutos.

Face a potencialidade do risco, surge uma nova inquietação e atenção por parte da comunidade científica: os resíduos nanotecnológicos. Com a preocupação sobre o nano lixo ou o nanowaste, apresenta o Jornal The Guardian o questionamento: Nano: uma Opção Mais Limpa? A Nanotecnologia Poderia Arrumar o Planeta sem Deixar Resíduos Perigosos? (tradução nossa). Para tais dúvidas não se vislumbram ainda respostas com certeza científica, mas pelo que se analisa dos estudos até agora apresentados, o nanowaste, ou resíduos nanotecnológicos, podem trazer mais riscos que os resíduos em escala macro (CAVE, 2013).

Atentos ao risco e estas características únicas das nanotecnologias, um ciclo adequado de avaliação dos nanomateriais - LCA - deve ser 
definido e conduzido muito antes de sua comercialização, para que seja considerado seguro. Adicionalmente, recursos apropriados devem ser destinados para pesquisar os processos, produtos e uso de materiais que impliquem em menor risco, uma vez que ocorrendo o descarte inadequado do nanowaste, enfrenta o ecossistema riscos sem precedentes e impactos ainda não vistos, sendo necessária a avaliação de todo ciclo de vida, da fabricação até o descarte, observando todas as particularidade desta manipulação em nanoescala, gerando alerta porque, mesmo em limitado número de estudos, estes apresentam os riscos de exposição (INTERNATIONAL CENTER FOR

\section{ASSESSMENT, 2007).}

Genericamente, o conceito de nanowaste pode ser entendido como aqueles materiais gerados a partir de um processo produtivo cuja ordem é do tamanho de nanômetros. Ou, ainda, os resíduos dos nanoprodutos, cuja vida útil tenha chegado ao seu fim e foram descartados. Dessa forma, a geração de nanowastes abrange duas cadeias: a) as indústrias na manipulação de nanomateriais e nanoprodutos decorrentes de seu sistema produtivo; e b) e os consumidores que também são responsáveis pela geração de nanowastes ao consumirem os nanoprodutos (MENDOZA; CABRERA, 2012).

Nos últimos anos, apesar de numerosas publicações e artigos sobre o crescimento exponencial da nanotecnologia global e suas particularidades, essas publicações contêm poucos dados científicos e abordagens viáveis de lidar com fluxos de nanowaste gerados em várias fases do ciclo de vida dos materiais. A falta de publicações científicas para gestão de fluxos de nanowaste é evidência de limitação ou um esforço limitado e concentrado dos investigadores neste domínio (MUSEE, 2011).

Nesta senda o conhecimento acerca do ciclo de vida dos nanomateriais é fundamental, pois a partir disto, viável promover a gestão adequada do risco na destinação final do nanowaste.

A gestão dos resíduos nanotecnológicos vem ganhando maior destaque na literatura internacional, face o risco do nanowaste no meio ambiente ser maior do que de materiais em escala macro, conforme observa a OECD:

\begin{abstract}
Um ponto em branco relativo no entendimento científico reside área de gerenciamento de resíduos. Os resíduos que contêm esses materiais são atualmente gerenciados juntamente com o desperdício convencional sem conhecimento suficiente dos riscos associados e Impactos no meio ambiente. (tradução nossa) (OCDE, 2016, p. 05).
\end{abstract}

A organização ainda esclarece que os processos atuais de gestão de resíduos e as técnicas não são geralmente apropriadas para abordar impactos potenciais de nanomateriais. Atuais instalações de tratamento de resíduos não são tipicamente projetadas para lidar com resíduos contendo nanomateriais, potencialmente levando a emissões para o meio ambiente e a exposição das pessoas a essas substâncias. Portanto, o estudo da OCDE visa identificar o status do conhecimento nessa área, as falhas de conhecimento, bem como as áreas em que os trabalhos futuros devem ser conduzidos em prioridade (OCDE, 2016). Este protocolo será apresentado poderia ser validado como instrumento regulatório, já que possui especificações e conclusões capazes de realizar a gestão do risco.

Tal aspecto demonstra a inserção na complexa tarefa de alcançar uma alternativa de regulação, para que seja possível realizar a gestão dos riscos na destinação final do nanowaste, minimizando os (possíveis) danos à vida humana e meio ambiente, caso não sejam tomadas medidas precaucionais.

Outro aspecto que tem gerado preocupação é o destino final destes materiais com nanotecnologia quando do fim de seu ciclo. Estes materiais podem não ser biodegradáveis e assim, 
permaneceriam no ambiente, interagindo com outros materiais. Este risco em potencial já está causando preocupação dos países em desenvolvimento para onde os resíduos contendo nanomateriais podem ser exportados (FAO/WHO, 2012). A avaliação do ciclo de vida está diretamente vinculada à avaliação do risco das nanopartículas, e vice-versa.

Em face do risco apresentado pelo nanowaste e pelos nanoprodutos, bem como a inadequação da destinação final, urge fomentar a adoção de marco regulatório específico. Esta investigação propõe que se adote o protocolo da OCDE, como instrumento autorregulatório apto para prevenção dos danos ao meio ambiente e vida humana.

\section{ALTERNATIVAS}

\section{(AUTO)REGULATÓRIAS PARA GESTÃo DO RISCO: FLEXIBILIZAÇÃO DO SISTEMA DO DIREITO?}

A nanociência e a nanotecnologia $(\mathrm{N} \& \mathrm{~N}), \mathrm{o}$ estudo e a aplicação dessa tecnologia, respectivamente, ganham espaço no cenário brasileiro a partir dos anos 2000, quando ocorreram as primeiras iniciativas de reunir pesquisadores que já atuavam ou que tinham interesse na área (FERNANDES; FILGUEIRAS, 2008). No entanto, a discussão da regulação, na ótica jurídica, ainda é recente, incipiente e disciplinar.

Em virtude da crescente demanda de produção em escala nanotecnológica, verifica-se que os pesquisadores e autoridades nacionais iniciaram um processo de conscientização e de pesquisa sobre tal nova tecnologia. Observa-se no cenário mundial o concreto debate sobre as nanotecnologias, como se demonstrou no primeiro ponto deste estudo. A partir destes movimentos, tais organismos acabam por elaborar diretrizes, protocolos ou instrumentos com potencial regulatório. Bastaria do Direito um esforço no sentido de buscar alternativas para validar tais normas, as quais no cenário complexo das nanotecnologias, são as únicas bases científicas que poderiam orientar seu desenvolvimento de maneira mais segura, minimizando o risco.

Convém mencionar, aqui, a questão do resíduo e a responsabilidade civil dos diversos integrantes da cadeia consumerista, já consagrados nos princípios da Política Nacional de Resíduos Sólidos (Lei no . 12.305/2010), no artigo $3^{\circ}$, XVII que menciona a responsabilidade compartilhada pelo ciclo de vida dos produtos, ou seja, o conjunto de atribuições individualizadas e encadeadas dos fabricantes, importadores, distribuidores e comerciantes, dos consumidores e dos titulares dos serviços públicos de limpeza urbana e de manejo dos resíduos sólidos, para minimizar o volume de resíduos sólidos e rejeitos gerados, bem como para reduzir os impactos causados à saúde humana e à qualidade ambiental decorrentes do ciclo de vida dos produtos, nos termos da referida lei. Importante pensar que com a reciclagem pode-se estar perpetuando o ciclo de vida de um nanomaterial, que entrará novamente em um novo ciclo, do berço ao túmulo.

Em 13 de abril de 2016, o Centro de Direito Ambiental Internacional (CIEL), a Organização de Cidadãos Europeus pelo Meio Ambiente, a Organização para Standards (ECOS), e o Instituto Oeko efetuaram a Publicação de uma Declaração das Organizações Européias sobre resíduos que contenham nanomateriais. Entre os principais aspectos abordados estão: a implementação de plena responsabilidade do produtor para garantir uma gestão segura de resíduos contendo nanomateriais manufaturados; a restrição dos movimentos transfronteiriços de resíduos que contenham certos nanomateriais manufaturados; a possibilidade de quantificação transparente e caracterização dos fluxos de resíduos que contêm nanomateriais fabricados através de registro público dos nanoprodutos na União Europeia; o 
estímulo a inovação em matéria de prevenção de resíduos; a promoção do desenvolvimento de tecnologias de reciclagem e descarte seguras e eficazes para os produtos que contêm nanomateriais manufaturados; o desenvolvimento e estabelecimento de critérios verificáveis de fim de resíduos para materiais recicláveis que contêm nanomateriais manufaturados; e ainda que os inovadores devem explorar como as propriedades avançadas de nanomateriais fabricados podem ser utilizados em apoio da economia sem a introdução de novos riscos ambientais ou agravar os já existentes (DECLARATION, 2017).

Atualmente, em relação às nanotecnologias e mais especificamente ao nanowaste vislumbrase um movimento transnacional de regulação, acompanhado da tendência da autorregulação como meio apto a prestar respostas adequadas a estas novas demandas e incertezas dos resíduos nanotecnológicos. Existem inúmeros estudos, protocolos e outros instrumentos com potencial regulatório, que podem cumprir com o papel de um marco regulatório, frente às atuais lacunas legislativas e omissão estatal. Considerando-se a complexidade das nanotecnologias e os rápidos avanços do sistema da Ciência frente a este tema, uma lei fechada e estanque não parece ser a solução para regular as demandas da era nanotecnológica.

Assim, destaca-se o protocolo da OCDE (2016), organização internacional que desenvolveu estudo específico com os principais tipos de tratamentos de resíduos, com possíveis respostas à gestão do nanowaste.

Com base nas inquietações apresentadas e inseridos num cenário iluminado pela Precaução, a hipótese que se apresenta é verificar a possibilidade de adoção do protocolo da OCDE como instrumento autorregulatório adequado para elaborar uma gestão mínima do risco do nanowaste, como alternativa do mundo jurídico frente às incertezas e lacunas do Direito.
Pariotti (2010) discorre sobre a autorregulação, sugerindo inclusive que a adoção destes instrumentos possa superar a antiga dicotomia entre público e privado, inserindo-se no contexto plural, remetendo a Teubner (2005):

Nesse sentido, é intrigante ver se os modelos
regulatórios em algum momento poderiam superar a
dicotomia entre privado e público, expressando a
distinção entre estado e sociedade em termos de
'policontexturalidade', isto é, a idéia de que várias
perspectivas sociais se refletem na lei, sem uma
distinção entre eles sempre sendo possível. Ser uma
maneira de superar a visão que permite espaço para
autorregulação encoraja o eclipse da autoridade
pública por interesses privados.(Pariotti, 2010, p.25-
26).

A organização internacional da OECD tem um papel de destaque em nível global nas pesquisas e elaboração de instrumentos com potencial autorregulatório na área das nanotecnologias, principalmente no objeto deste artigo, nanowaste e autorregulação.

Este movimento autorregulatório efetua uma comunicação entre sistemas diversos, sendo inevitável tal interação no cenário das nanotecnologias, que neste sentido propõe ainda a interdisciplinaridade. Seriam sistemas diferentes trabalhando em sintonia, unindo esforços onde é possível a consonância de informações e estudos.

A fim de embasar a adoção de mecanismos alternativos de regulação, importante entender sobre as diferenças entre meta e autorregulação.

Para Berger Filho (2016, p. 24), a autorregulação representa "[...] novas formas de operar o Direito na globalização [...]" e "resultam de regras, acordos e regulação de atores privados - empresas transnacionais - que através do fluxo de suas atividades ao redor do mundo, proporcionam a difusão destas normas".

A autorregulação é:

[...] a proliferação de normas estabelecidas por organizações profissionais e de padronização técnica, a ampliação das declarações de princípios, 
orientações e diretrizes estabelecidas por organizações internacionais relativas a demandas globais, das quais os Estados não conseguem formar consenso para imposição de obrigações concretas, resultam na ascensão de instrumentos de soft law. Normas brandas e flexíveis, cujo descumprimento não acarreta nenhum tipo de sanção formalmente instituída pelo Estado (ou Estados no caso do Direito Internacional). (BERGER FILHO, 2016, p. 24).

Em outras palavras, sobre autorregulação:

Hoje é fundamental ter em conta o fenômeno da autorregulação para elaborar os critérios de imputação coerentes com a nova realidade social e jurídica do Estado. Com efeito, na era do risco, os avanços técnicos estabeleceram um conflito fundamental entre sociedade, Estado e empresa. Assim, o Estado, na era da incerteza, perdeu o monopólio tanto da proteção de bens jurídicos, como da distribuição de riscos e do planejamento estratégico, uma vez que simples e cheio, necessitava de informações suficientes para poder desenvolvê-los. Isso se vê claramente na matéria ambiental na qual o Estado já não dispõe do conhecimento e de recursos para poder regular corretamente os novos riscos ambientais.(DIEZ, 2013, p.16).

Ante as inovações da nanotecnologia e sua necessidade de regulação, esta tem assumido um significado mais amplo, abarcando também instrumentos de metarregulação, conforme lecionam Engelmann, Aldrovandi e Berger Filho (2013). Apesar de não existir definição consensual de autorregulação e metarregulação, de maneira geral pode-se dizer que a primeira é um processo de regulação voluntária, instituído independentemente da regulamentação estatal, criada por atores não governamentais, inclusive por seu(s) próprio(s) destinatário(s). Já a metarregulação resulta da interação entre a regulação estatal e a autorregulação. Ela seria uma estratégia de supervisão do Estado sobre os mecanismos da autorregulação (ENGELMANN; ALDROVANI; BERGER FILHO, 2013).
É preciso lembrar que hoje não existem legislações acerca do nanowaste e que dificilmente uma norma elaborada pelo legislativo estaria de acordo com as necessidades e complexidades nanotecnológicas. Mas, ao contrário de toda a inércia estatal, já pode-se contar com alguns instrumentos com potencial para regulação, de organismos internacionais, tanto governamentais como privados, que auxiliariam no fornecimento de protocolos e regulações específicas. Tais documentos são fruto de extensos e profundos estudos relacionados à nanotecnologia, segurança e nanorresíduos (LEAL; ENGELMANN, 2018, p.141).

Desta forma, a adoção de uma postura não inerte por parte do Direito, em busca de um autorregulação pode ser uma das alternativas viáveis e palpáveis frente aos desafios nanotecnológicos. Busca-se respeitar a sustentabilidade e preservação dos direitos das futuras gerações. A autorregulação vem se mostrando atual, efetiva e adequada ao contexto das nanotecnologias e a necessária adoção de regulação específica:

\begin{abstract}
A autorregulação através do cumprimento de documentos regulatórios das agências reguladoras e organizações de normalização técnica, pode atender aos requisitos de proteção ao ser humano e meio ambiente, utilizando a comunicação como o norte a ser seguido em todas as etapas dos processos internos, serviços prestados e produto (levando-se em conta as cláusulas contidas em convenção coletiva por exemplo; considerando-se também a questão da rotulagem dos produtos e o direito à informação) (MARTINS, 2016, p. 90).
\end{abstract}

O Direito, através do movimento estatal e legislativo, mostra-se insuficiente, e vem, ao longo do tempo, perdendo espaço para outras áreas do saber que estão se permitindo fazer regulação. Assim, cabe a observação de Marchant e Sylvester (2016, p.715): 
A quietude da academia jurídica é, cremos, um erro. Compreender a interação da regulação e a tecnologia é uma área onde os juristas e os profissionais têm muito a oferecer. Na verdade, acreditamos que a lei e, em particular, os quadros legais (frameworks) e princípios aplicados em outras áreas do desenvolvimento tecnológico, podem destacar os riscos e benefícios de regulações da nanotecnologia. Em particular, acreditamos que a experiência de agências reguladoras internacionais, estruturas e arranjos provarão áreas frutíferas de pesquisa na exploração de nanotecnologias para uma regulação futura.

No caso da autorregulação do nanowaste, com adoção do protocolo da OCDE baseado em critérios científicos, oriundos de pesquisa específica sobre resíduos nanotecnológicos, fomentaria o desenvolvimento responsável das nanotecnologias, promovendo uma verdadeira gestão do risco, administrando adequadamente a destinação final do nanowaste, com uma preocupação voltada à sustentabilidade. (LEAL; ENGELMANN, 2018, p.158).

Esta flexibilização por parte do sistema do Direito seria baseada nos fundamentos do Pluralismo Jurídico de Teubner (2005). Teubner (1989, p.125) entende que a convergência de expectativas pode ser estimulada com a "[...] introdução voluntária de mecanismos de regulação [...]" que permitem adentrar "No domínio de uma regulação da coevolução, onde sistemas de negociação intersistêmicos são desenhados com o objetivo precípuo de compatibilizar mundividências e expectativas convergentes" (TEUBNER, 1989, p.125).

Observa-se o desafio de promover uma adaptação e uma aprendizagem de cada sistema diante do aumento expressivo da fragmentação e dos processos de diferenciação funcional das mais diferentes organizações.

Fazendo uma ligação entre a construção acima e o estudo em tela, cabível a tese do autor à ausência de regulação do nanowaste e demais áreas da nano, pois a OECD, com sua participação em nível internacional, faria a comunicação, apresentando seus estudos específicos, neste caso o protocolo de gestão do nanowaste, acoplando tal protocolo ao mundo jurídico, que seria irritado para adotar tais recomendações. Vale ressaltar que não somente as empresas poderiam adotar tal autorregulação, mas o próprio Estado poderia acoplar tal estrutura, de um sistema diverso, fazendo jus à comunicação e flexibilidade estrutural imposta neste contexto (LEAL; ENGELMANN, 2018, p. 209).

Ademais, possível a ligação da autorregulação do nanowaste nesta doutrina, ressaltando quando Teubner $(2003$, p.11) diz que "[...] o direito global só pode ser interpretado adequadamente por meio de uma teoria do pluralismo jurídico e de uma teoria das fontes do direito, correspondentemente concebida em termos pluralistas".

O sistema do Pluralismo Jurídico entendido como o movimento que valida normas estranhas ao Direito, adotando a postura de que o Estado não é a fonte única e exclusiva de juridicidade, destacando-se a produção normativa gerada por grupos organizados semi autônomos que compõem a tessitura social e pressupondo-se que apenas uma pequena parcela das inúmeras expectativas que regem a vida social, e não necessariamente as mais importantes delas para todos os grupos, encontram guarida no Direito Positivo Estatal - reforça a importância e utilização de normas informais, de outras esferas e organizações, resolvendo-se assim o problema de ausência de normas estatais, que na atual sociedade pós-moderna sequer resolveriam as demandas dotadas de complexidade:

O pluralismo jurídico fascina os juristas pósmodernos, que não se preocupam mais com o direito oficial do Estado centralizado e suas aspirações de abstração, generalidade e universalidade. É na 'lei do asfalto' das grandes cidades norte-americanas ou no 'quase direito' das favelas do Brasil, nas normas informais das culturas políticas alternativas, na colcha de retalhos do direito das minorias, nas 
normas dos grupos étnicos, culturais e religiosos, nas técnicas disciplinares da 'justiça privada' e, ainda nos regulamentos internos de organizações formais e redes informais que se encontram todos os ingredientes da pós-modernidade: o local, o plural, o subversivo. A diversidade dos discursos fragmentados e hermeneuticamente fechados pode ser identificada por meio de numerosos tipos informais de regras, geradas quase independentemente do Estado e operando em várias esferas informais. O pluralismo jurídico descobre, assim, no 'lado obscuro' do direito soberano, o potencial subversivo dos discursos reprimidos. As mais diversas quase-normas informais e locais são tidas como supplément ao moderno ordenamento jurídico oficial, formal, centralizado. Exatamente esta ambivalência, esse caráter dúplice, faz o pluralismo jurídico tão atraente aos olhos dos juristas pós-modernos (TEUBNER, 2005, p.81).

Desta forma, mostra-se possível a adoção do protocolo da OCDE, como instrumento autorregulatório para a gestão do nanowaste, cumprindo com os critérios precaucionais que são imprescindíveis a uma adequada gestão dos riscos nanotecnológicos. A adoção de mecanismo autorregulatório é então, uma possível alternativa viável frente às complexidades dos riscos nanotecnológicos e as novas exigências provindas deles. O movimento estatal, através de processo legislativo, não acompanha a velocidade exigida pelas nanotecnologias.

Com um consumo cada vez maior, aumenta-se também o descarte de embalagens e mesmo de produtos ou de resíduos dos produtos com nanotecnologia junto ao meio ambiente, sendo necessário que se implementem algumas medidas em relação a essa nova realidade.

Assim, ao utilizar-se do que há de mais aprofundado e referência no campo de resíduos nanotecnológicos, como as diretrizes geradas pela OCDE, demonstra-se uma tentativa prática de se atender ao princípio da precaução, da sustentabilidade e da equidade intergeracional. Tal princípio, inclusive, gera divergências quanto sua aplicação, dimensão e definição, mas levando-se em consideração a complexidade desta nova tecnologia, o mesmo pode ser utilizado.

Este importante princípio ambiental surgiu originalmente na Conferência da Organização das Nações Unidas (ONU) sobre Meio Ambiente realizada em Estocolmo, na Suécia, em 1972. Foi primeiramente incorporado por um ordenamento jurídico nacional na Alemanha denominado de Vorsorgeprinzip, o qual passou a nortear as políticas ambientais alemã. Foi aplicado, posteriormente, na Conferência Internacional sobre a Proteção do Mar do Norte, em 1984 e na Convenção de Viena, ano de 85, surgindo como princípio autônomo em 1987, na segunda edição da Conferência Internacional sobre a Proteção do Mar do Norte. No âmbito da Comunidade Europeia, passou a integrar o rol de princípios comunitários através do Tratado de Maastricht. Na Conferência das Nações Unidas sobre Meio Ambiente e desenvolvimento, no Rio de Janeiro (Rio 92), o princípio da precaução passou a integrar o rol de princípios produzidos naquela oportunidade juntamente com outros princípios fundamentais para o desiderato de promover a proteção do meio ambiente (WEYERMULLER, 2010).

Defende Pariotti (2010, p. 18) sobre a construção de instrumento regulamentar para as nanotecnologias, ante o cenário de incertezas, com adoção do princípio da precaução: A construção de um quadro regulamentar para
tecnologias emergentes, no entanto, requer duas
etapas preliminares, respectivamente, tratando de
esclarecer princípios de fundo e abraçando um
modelo de gerenciamento de risco específico. A fim
de conceber uma abordagem regulamentar das
nanotecnologias, primeiro deve ser perguntado se,
do ponto de vista jurídico, a noção de risco difere da
noção de incerteza. Parece que isso difere, o que é a
razão do princípio da precaução, embora
questionável no seu significado e implicações, dever
ser aplicado. Os reguladores precisam saber se os
nanoprodutos são potencialmente prejudiciais, mas 
eles têm que regular mesmo quando os riscos e o dano não são quantificáveis. (tradução nossa).

Possível acrescentar a visão de Tallacchini (2014, p.87) sobre a precaução e incerteza da ciência, adequando-se ao cenário de risco do nanowaste:

A crescente dimensão da incerteza no conhecimento científico, particularmente no campo das novas ciências da vida, são os fenômenos que estão cimentando uma visão diferente das relações entre ciência e sociedade. Com a expressão "incerteza da ciência", faz-se referência a várias formas de indeterminações do conhecimento no campo científico: a complexidade do conhecimento, a falta ou insuficiência de dados, a imprevisibilidade dos sucessos, o caráter estocástico das previsões em muitos setores.

Portanto, necessário o suporte à gestão dos riscos enfrentados na sociedade pós-moderna conhecendo e aplicando minimamente os elementos estruturantes do Princípio da Precaução, que pode trazer fundamento e lastro para a efetivação da autorregulação dos nanomateriais e sua correta destinação. É preciso que haja uma antecipação aos resultados negativos de nossa ação modificadora através da aplicação do princípio da precaução, o qual se liga à necessidade de uma comunicação ambientalmente relevante entre os sistemas sociais do Direito e da Economia para atingir o benefício do meio ambiente. Precisa-se de uma comunicação em que se realize uma espécie de ponderação entre os interesses coletivos (meio ambiente preservado) e as pressões de uma economia (WEYERMULLER, 2010), conflito este presente na era nanotecológica.

Importante destacar que nas negociações internacionais na área ambiental, o princípio da precaução ou cautela é cada vez mais invocado, motivando esforços para a institucionalização de instrumentos reguladores, dirigidos para garantir o que já se convencionou denominar de biossegurança e para o controle dos movimentos transfronteiriços de organismos geneticamente modificados, como atesta o firmado Protocolo Internacional de Biossegurança (janeiro de 2000), mas que diz respeito às segurança biológica (ALBAGLI, 2001).

Contudo, ressalvas a este princípio devem ser realizadas, apontando certas fragilidades, as quais originam críticas à precaução. Alertando quanto à utilização deste princípio, Douglas e Wildavsky (2012) afirmam que o uso mecânico da precaução pode obscurecer o ponto central na discussão sobre os riscos, uma vez que não há maneira de evitar o risco em vida. Em seu entendimento a saída é a utilização de todo conhecimento disponível para distinguir os riscos grandes, os bem estabelecidos, aqueles que são prováveis e aqueles que as evidências sugerem ser insignificantes ou inexistentes. Por outro lado, ainda existem posições divergentes na doutrina quanto a efetividade do Princípio da Precaução, principalmente no sentido de haver inúmeras limitações a este instrumento ambiental, como a ambiguidade inerente do princípio; a cobertura do princípio somente para determinados tipos de riscos; e percepção diferente de cada tomador de decisão quanto ao risco, que dificulta sua aplicação, principalmente se levado em consideração o modelo econômico de interpretação da precaução na gestão do risco. Ademais, apresentam-se tantos problemas teóricos quanto de coleta de dados para aplicabilidade, gerando inconsistência de parâmetro a ser aplicado em sistemas de gestão, de forma ainda que a precaução não pode gerar decisões singulares, o que demanda a multidisciplinariedade e responsabilidade das áreas política, jurídica, econômica (GONÇALVES, 2013).

Tem-se que, por ser um instrumento de ação, tal princípio gera novos deveres aos entes públicos e privados, mas conforme já mencionado, ela levanta questionamentos, ante 
sua ambiguidade, imprecisão, o que gera a resistência ou até mesmo dificuldade de aplicação. Possibilitando o desenvolvimento de mecanismos para sua aplicação, viável seria utilizar-se do que dispõe a comunicação de âmbito geral da Comissão Europeia de 2000 sobre o princípio da precaução, por exemplo, como análise de riscos, critérios de sua aplicação, dentre outros Para tanto, deve a precaução acudir a uma abordagem estruturada, uma avaliação científica, tão completa quanto possível, dos riscos para o ambiente e/ou a saúde a fim de identificar os resultados objetivos existentes, as lacunas de conhecimento e as incertezas científicas. Feito isso, poderá ser selecionado o mais adequado plano de ação. Segundo a Comissão, os quatro componentes que devem fazer parte da avaliação científica são: a) identificação do perigo; b) caracterização do perigo; c) avaliação da exposição; e d) caracterização do risco (COMISSÃO DAS COMUNIDADES EUROPEIAS, 2000).

Assim, muito embora existam dificuldades na aplicabilidade e definição de tal princípio, o mesmo precisa ser considerado nas tomadas de decisões dotadas no cenário de complexidade, principalmente tratando-se das nanotecnologias, que já apresentam evidências de risco, de maneira que, caso não adotada cautela, poderiam comprometer a presente e futuras gerações.

\section{CONCLUSÃO}

A sociedade atual está vivendo o período da revolução nanotecnológica, onde os mais diversos setores produtivos utilizam em seus processos insumos, materiais ou equipamentos que contém nanopartículas produzidas em meio industrial. As pesquisas em nano escala já deixaram os laboratórios e ganharam as linhas de produção das indústrias, incrementando o número de produtos com nanotecnologia que chegam ao mercado.
Atualmente enfrentam-se novos desafios, que ultrapassam as barreiras dos laboratórios, atingindo as incertezas científicas e riscos no que se refere aos impactos no meio ambiente e vida humana.

A recente preocupação com as consequências das nanotecnologias vem despertando uma inquietude iminente no que tange à proteção do meio ambiente, incluindo-se a vida humana. Pelo que se depreende do estudo de vasta bibliografia e notícias científicas recentes, a produção em nano escala encontra-se em amplo crescimento, o que consequentemente gera o aumento de depósito e descarte de resíduos com nanomateriais.

Um aspecto que tem gerado preocupação para a comunidade científica é o nanowaste, de que maneira este novo resíduo poderá afetar o ecossistema se os estudos publicados sobre seus potenciais riscos se confirmarem? Não há certeza sobre os (possíveis) danos que pode causar o descarte deste nanowaste no meio ambiente, o que reflete na existência do Risco, necessitando-se assim de umaalternativa a fim de proporcionar a destinação final adequada a estes nanomateriais.

Assim, frente a estes novos riscos das nanotecnologias, cabe ao Direito buscar apresentar uma alternativa jurídica que efetive a regulação dos novos produtos, em especial do nanowaste. Neste sentido, apresenta-se como meio jurídico apto o protocolo da OCDE, estudo específico sobre os tratamentos de resíduos com nanopartículas. Atrelado a esta orientação, apresenta-se a evolução do debate e conclusões pertinentes a estas diretrizes, que fomentam ainda mais a regulação, seguindo uma tendência de modelo autorregulatório de regulação das nanotecnologias.

Não se pode esquecer que há uma necessidade urgente de se discutir a postura do Direito frente a estas novas questões, lembrando que uma alternativa é que este ramo do conhecimento considere sempre em suas decisões 
sobre riscos o princípio da precaução, com uma fundamentação baseada na melhor técnica disponível, nas boas práticas e fazendo uso do diálogo entre as fontes nacionais e internacionais, de origem estatal ou não, de modo a garantir a geração de desenvolvimento e não de danos.

A fim de fundamentar esta validação ou adoção de normas "quase-direito" - como normativas de organizações não governamentais, protocolos ou diretrizes técnicas, não estataisparafraseando Teubner (2005), importante fundamentar tal movimento através das bases do pluralismo jurídico, levando em consideração que as diretrizes hoje existentes ao redor do mundo sobre nano são elaboradas por outras ciências. Tal flexibilização ainda faria jus à interdisciplinaridade que esta nova tecnologia demanda.

As nanotecnologias provocarão como legado, no Direito, uma revolução, uma vez que exigirão a tomada de posicionamentos jurídicos e não apenas legalistas, ou seja, exigirão inovação no/do Direito.

\section{REFERÊNCIAS}

ALBAGLI, Sarita. Biodiversidade e biotecnologia na geopolítica e na geoeconomia mundiais. In: Centro Internacional de Desenvolvimento Sustentável. Comércio e meio ambiente. Brasília: Ministério do Meio Ambiente, 2001.

BARBAT, A. S. Estudios de Derecho de Seguros y Reaseguros. Uruguai: La Ley, Montevideo, 2016.

BECK, Ulrich. La Sociedad del Riesgo Mundial: en busca de la seguridad perdida. Traducción de Rosa S. Carbó. Barcelona: Paidós, 2008.

BERGER FILHO, Aírton Guilherme. A governança dos riscos das nanotecnologias e o princípio da precaução: um estudo a partir da teoria dialética da rede. Tese (Doutorado em Direito) - Programa de Pós-Graduação em Direito, Universidade do Vale do Rio dos Sinos (UNISINOS), São Leopoldo, 2016. Disponível em: <http://www.repositorio.jesuita. org.br/bitstream/handle/UNISINOS/5563/A\%c3\%adrton\%20Guilherme\%20Berger\%20Filho2_pdf?s equence $=3 \&$ isAllowed $=\mathrm{y}>$. Acesso em: 26 jun. 2018.

BRASIL. Lei $n^{\circ}$ 12.305, de 2 de agosto de 2010. Institui a Política Nacional de Resíduos Sólidos; altera a Lei no 9.605, de 12 de fevereiro de 1998; e dá outras providências. Disponível em: <http://www.planalto.gov.br/ccivil_03/_ato2007-2010/2010/lei/112305.htm>. Acesso em: 24 jun. 2018.

BRITTO, Roberta Socoowski et al. Effects of carbon nanomaterials fullerene C60 and fullerol C60 (OH) 18-22 on gills of fish Cyprinus carpio (Cyprinidae) exposed to ultraviolet radiation. Aquatic Toxicology, Amsterdam, v. 114-115, jun. 2012. Disponível em: <http://www.sciencedirect.com/science/article/pii/ S0166445 X12000689>. Acesso em: 07 nov. 2018.

CAVE, Holly. Nano: a cleaner option? Could nanotechnology tidy up the planet without leaving a dangerous residue of its own?. The Guardian, London, Oct. 18 2013. Disponível em: <https://www.the guardian.com/what-is-nano/nano-a-cleaner-option?CMP=share_btn_link>. Acesso em: 05 jun. 2018.

CHRISTENSEN, Frans Møller et al. Better control of nanomaterials: summary of the 4-year Danish iniciative on nanomaterials. Copenhagen: The Danish Environmental Protection Agency, 2015. 
(Environment protect, n. 1797). Disponível em: <http://www2.mst.dk/Udgiv/publications/ 2015/12/978-87-93352-89-6.pdf>. Acesso em: 08 jun. 2018.

COMISSÃO DAS COMUNIDADES EUROPEIAS. Comunicação da comissão: relativa ao princípio da precaução. 2.2.2000 COM (2000) 1 final. Bruxelas, 2000. Disponível em: <http://eur-lex.europa. eu/legal-content/PT/TXT/?uri=celex:52000DC0001>. Acesso em: 05 ago. 2017.

DAL FORNO, Gonzalo Ogliari et al. Intraperitoneal exposure to nano/microparticles of fullerene (c60) increases acetylcholinesterase activity and lipid peroxidation in Adult Zebrafish (Danio rerio) Brain. BioMed Research International, New York, v. 2013, 2013. Disponível em: <https://www.hindawi.com/journals/ bmri/2013/623789/abs/>. Acesso em: 10 nov. 2018

DECLARATION on Waste Containing Nanomaterials. Disponível em: $<$ http://www.natlawreview.com/article/european-organizations-issue-declaration-waste-containingnanomaterials\#sthash.k6eBXpMK.S2cTdme9.dpuf. >. Acesso em: 10 nov. 2018.

DELMAS-MARTY, Mireille. Libertés et sûreté dans un monde dangereux. Paris: Éditions du Seuil, 2010

DÍEZ, Carlos Gómez-Jara. A responsabilidade penal da pessoa jurídica e o dano ambiental: a aplicação do modelo construtivista de autorresponsabilidade. A Lei 9605/98. Porto Alegre: Livraria do Advogado Editora,2013.

DOUGLAS, Mary; WILDAVSKY, Aaron. Risco e cultura: um ensaio sobre a seleção dos riscos tecnológicos e ambientais. Tradutora Cristiana de Assis Serra. Rio de Janeiro: Elsvier, 2012

ENGELMANN, Wilson. Os avanços nanotecnológicos e a (necessária) revisão da Teoria do Fato Jurídico de Pontes de Miranda: compatibilizando "riscos" com o "direito à informação" por meio do alargamento da noção de "suporte fático". In: CALLEGARI, André Luís; ROCHA, Leonel Severo; STRECK, Lenio Luiz (orgs.) Constituição, sistemas sociais e hermenêutica: anuário do programa de Pós-graduação em Direito da UNISINOS: mestrado e doutorado. Porto Alegre: Livraria do Advogado Editora; São Leopoldo: UNISINOS, 2011. p. 339-363.

ENGELMANN, Wilson; ALDROVANDI, Andrea; BERGER FILHO, Airton Guilherme. Perspectivas para a regulação das nanotecnologias aplicadas a alimentos e biocombustíveis. Vigilância Sanitária em Debate: Sociedade, Ciência \& Tecnologia, Rio de Janeiro, v. 1, n. 4, nov. 2013. Disponível em: <https://visaemdebate.incqs.fiocruz.br/index.php/visaemdebate/article/view/69/ 76>. Acesso em: 11 ago. 2017.

FAO/WHO-. Food and Agriculture Organization of the United Nations/World Health Organization, Seminar on Nanotechnologies in food and agriculture Roma, 27 Mar. 2012. Disponível em: <http://www.fao.org/fileadmin/user_upload/agns/news_events/Nano_Seminar_Agenda_FINAL.pdf>. Acesso em: 07 jul. 2018.

FERNANDES, Maria Fernanda Marques; FILGUEIRAS, Carlos A. L. Um panorama da nanotecnologia no Brasil (e seus macro-desafios). Química Nova, São Paulo, v. 31, n. 8, 2008. Disponível em: <http://quimicanova.sbq.org.br/imagebank/pdf/Vol31No8_2205_49-AG07508.pdf>. Acesso em: 28 jun. 2017. 
GAVANKAR, Sheetal; SUH, Sangwon Suh; KELLER, Arturo F. Life cycle assessment at nanoscale: review and recommendations. The International Journal of Life Cycle Assessment, n. 17 p. 295-303, mar. 2012.

GONÇALVES, Vasco. Critical approach of the use of economic models in precaucionary risk management. European Journal on Risk Regulation: EJRR, Berlin, v. 4, n. 3, 2013. Disponível em: <https://www.jstor.org/stable/24323403?seq=1\#page_scan_tab_contents>. Acesso em: 07 nov. 2018.

HIGH-LEVEL Expert Group on Key Enabling Technologies. Final Report, June 2015: KETs: Time to Act, European Commission. Disponível em: <http://ec.europa.eu/growth/industry/key-enablingtechnologies/european-strategy/high-level-group/index_en.htm> Acesso em: 09 ago. 2017.

HOHENDORFF, Raquel Von. A Contribuição do Safe by design na estruturação autorregulatória da gestão dos riscos nanotecnológicos: Lidando com a Improbabilidade da Comunicação Inter-Sistêmica entre o Direito e a Ciência em Busca de Mecanismos para Concretar os Objetivos de Sustentabilidade do Milênio. 2018. Tese (Doutorado em Direito) Programa de Pós-graduação Mestrado e Doutorado, Universidade do Vale do Rio dos Sinos, São Leopoldo, 2018.

HOLLINGWORTH, P. The light and fast organization: a new way of dealing with uncertainty. Melbourne: Wiley, 2016.

HORIZON 2020 em breves palavras: O programa-quadro de investigação e inovação da EU. Disponível em: <https://ec.europa.eu/programmes/horizon2020/sites/horizon2020/files/H2020_PT_KI0213413PTN.p df> Acesso em: 09 ago. 2017.

INTERNATIONAL Center for Technology Assessment. Princípios para a supervisão de nanotecnologias e nanomateriais. Traduzido por Secretaria Regional Latino-Americana da União Internacional dos Trabalhadores na Alimentação, Agricultura, Hotelaria, Restaurantes, Tabaco e Afins (Rel-UITA). Washington: Nanoaction: International Center for Technology Assessment, jan. 2007. (NanoAction Project). Disponível em: $\quad$ http://www6.reluita.org/nanotecnologia/Principios_Supervision_ NANOTECNOLOGIAS-por.pdf >. Acesso em: 05 jun. 2018.

INTRODUCTION. Nanotechnology Products Database (NPD).[S.1.], 2018. Disponível em: <http://product.statnano.com/>. Acesso em: 07 jul. 2018

ISO - International Organization for Standardization. Ballot on ISO/DTR 12885 - Nanotechnologies Healthandsafety practices in occupationalsettings. Relatório Técnico, 2017.

LEAL, Daniele Weber S. ENGELMANN, Wilson A autorregulação da destinação final dos resíduos nanotecnológicos (NANOWASTE): o Pluralismo Jurídico entre a gestão dos riscos e os protocolos da OECD. São Leopoldo: Karywa, 2018.

LUHMANN, Niklas. El derecho de la sociedad. 2. ed. Herder: Ed. Universidad Iberoamericana, 2005. . Sociedad y sistema: la ambición de la teoria. Traducción de Santiago López Petit y Dorothee Schmitz. Barcelona: Paidós, 1990. 
MARCHANT, Gary E; SYLVESTER, Douglas J. Transnational models for regulation of nanotechnology. The Journal of Law, Medicine \& Ethics, Boston, v. 34, n. 4, p. 715, 2006. Disponível em: <http://onlinelibrary.wiley.com/doi/10.1111/j.1748-720X.2006.00091.x/abstract>. Acesso em: 25 jun. 2018.

MARTINS, Patrícia Santos. O sistema de normas ISO e as nanotecnologias: as interfaces regulatórias e o diálogo entre as fontes do Direito. 2016. Dissertação (Mestrado em Direito) - Programa de PósGraduação em Direito, Universidade do Vale do Rio dos Sinos (UNISINOS), São Leopoldo, 2016. Disponível em: <http://www.repositorio.jesuita.org.br/ handle/UNISINOS/ 6206>. Acesso em: 13 jul. 2017.

MENDOZA, Carlos Felipe; CABRERA, Laura Meraz. Hacia la nanociencia verde nanomateriales, nanoproductos y nanorresiduos. Materiales Avanzados, México, ano 10, n. 19, ago. 2012. Disponível em: <http://www.iim.unam.mx/revista/pdf/numero19.pdf>. Acesso em: 05 jun. 2018.

MUSEE, N. Nanowastes and the environment: potential new waste management paradigm. Environment International, v. 37, n. 1, jan. 2011. Disponível em: <http://www.sciencedirect.com/ science/article/pii/S0160412010001558>. Acesso em: 05 jun. 2018.

ORGANISATION for Economic Co-Operation and Development (OECD). Nanomaterials in waste streams: current knowledge on risks and impacts. Paris, 2016. Disponível em: <http://dx.doi.org/10.1787/ 9789264249752-en>. Acesso em: 24 jun. 2018.

PARIOTTI, Elena. Law, uncertainty and emerging technologies: towards a constructive implementation of the precautionary principle in the case of nanotechnologies. Persona y Derecho, Pamplona, n. 62, 2010. Disponível em: <http://heinonline.org/HOL/LandingPage? handle=hein.journals/persodcho62\&div=5\&id=\&page=>. Acesso em: 05 ago. 20187.

SCHWAB, Klaus. A quarta revolução industrial. Tradutor Daniel Moreira Miranda. São Paulo: Edipro, 2016.

SOM, Claudia et al._The importance of life cycle concepts for the development of safe nanoproducts. Toxicology, n. 269, vol. 2-3, p. 160-169, mar. 2010.

TALLACCHINI, Mariachiara. Principio de precaución y bioseguridad: aplicación a la salud humana. In: ROMEO CASABONA, Carlos Maria (Ed.). Principio de precaución, biotecnología y derecho. Granada: Comares, 2004.

TEUBNER, Gunter. A Bukowina global sobre a emergência de um pluralismo jurídico transnacional. Impulso, Piracicaba, v. 14, n. 33, p. 11, 2003. Disponível em: <http://livrozilla.com/ doc/1623775/abukowina-global-sobre-a-emerg\%C3\%AAncia-de-um-pluralismo>. Acesso em: 15 jun. 2018.

Direito, sistema e policontexturalidade. Apresentação: Dorothee Susanne Rüdiger. Introdução: Rodrigo Octávio Broglia Mendes. Piracicaba: Ed. UNIMEP, 2005.

O direito como sistema autopoiético. Tradução de José Engracia Antunes. Lisboa: Fundação Calouste Gulbenkian, 1989.

WEYERMÜLLER, André Rafael. Direito ambiental e aquecimento global. São Paulo: Atlas, 2010. 
Recebido em: 08/07/2018 Aceito em: 27/11/2018 
